Proceeding Paper

\title{
Microwave-Assisted Asinger Synthesis of Thiazolines ${ }^{\dagger}$
}

\author{
Raúl Eduardo Gordillo-Cruz 1,2, Liliana Gonzalez-Reyes 1,2, Milton Coporo-Reyes 1, \\ Nieves Zavala-Segovia 1,3, Bernardo A. Frontana-Uribe 1,3, Marco A. García-Eleno 1,2, \\ M. V. Basavanag Unnamatla ${ }^{1,2}$ and Erick Cuevas-Yañez ${ }^{1,2, *}$
}

Citation: Gordillo-Cruz, R.E.; Gonzalez-Reyes, L.; Coporo-Reyes, M.; Zavala-Segovia, N.; Frontana-Uribe, B.A.; García-Eleno, M.A.; Unnamatla, M.V.B.; Cuevas-Yañez, E. Microwave-Assisted Asinger Synthesis of Thiazolines. Chem. Proc. 2021, 3, 27. https://doi.org/10.3390/ ecsoc-24-08316

Academic Editors: Julio A. Seijas and M. Pilar Vázquez-Tato

Published: 14 November 2020

Publisher's Note: MDPI stays neutral with regard to jurisdictional claims in published maps and institutional affiliations.

Copyright: $\odot 2020$ by the authors. Licensee MDPI, Basel, Switzerland. This article is an open access article distributed under the terms and conditions of the Creative Commons Attribution (CC BY) license (http://creativecommons.org/licenses/by/4.0/).
1 Centro Conjunto de Investigación en Química Sustentable UAEM-UNAM, Carretera Toluca-Atlacomulco Km. 14.5, Toluca, Estado de México 50200, Mexico; rulis24@gmail.com (R.E.G.-C.); lili.gor07@gmail.com (L.G.-R.); mailto:mcopororeyes@gmail.com (M.C.-R.); nievesz@unam.mx (N.Z.-S.); bafrontu@unam.mx (B.A.F.-U.); magarciae@uaemex.mx (M.A.G.-E.); mvbasavanagu@uaemex.mx (M.V.B.U.)

2 Facultad de Química, Facultad de Química, Universidad Autónoma del Estado de México, Paseo Colón esq. Paseo Tollocan, Toluca 50120, Mexico

3 Instituto de Química, Universidad Nacional Autónoma de México, Circuito Exterior S. N., Ciudad Universitaria, Coyoacán, Ciudad de México 04510, Mexico

* Correspondence: ecuevasy@uaemex.mx; Tel.: +52-722-276-6610 (ext. 7734); Fax: +52-722-217-5109

+ Presented at the 24th International Electronic Conference on Synthetic Organic Chemistry, 15 November-15 December 2020; Available online: https://ecsoc-24.sciforum.net/.

Abstract: An array of 2,4-disubstituted thiazolines was obtained through Asinger reaction approach from the straightforward treatment of diverse aldehydes/ketones with 1-mercaptopropan-2-one, in the presence of $\mathrm{NH}_{3}$ assisted by microwave irradiation, displaying similar and sometimes higher yields, as well as shorter reaction times that traditional Asinger reaction conditions at room and lower temperatures.

Keywords: thiazoline; Asinger reaction; microwave irradiation

\section{Introduction}

Thiazolines are five-membered heterocycles that contain both nitrogen and sulfur, belonging to 1,3-azole heterocycles group. Compared with other azole rings, the thiazoline moiety is relatively less studied, in spite of the growing interest raised by this heterocycle, as a component of many natural products and synthetic compounds displaying a wide range of biological activities [1]. In this regard, diverse synthetic protocols for thiazolines were developed from condensations of thioamides and derivatives [2-6], as well as cysteine and other amino acids [7-9].

On the other hand, since its discovery in the late fifties [10-12], Asinger reaction showed the distinctive advantages provided by multicomponent reactions applied to the synthesis of cyclic compounds [13-17]. However, these apparent benefits were missed probably due to the few starting materials reported in the literature, which are compatible (and sometimes soluble) with aqueous ammonia, in addition to reaction temperatures close to ambient conditions or lower [18,19].

These facts motivated us to explore alternative conditions for the Asinger Reaction, in order to incorporate structurally diverse feedstock, keeping the attractive characteristics of this process and inherent multicomponent reaction approach. In this report, we disclose our first findings in this area.

\section{Results and Discussion}

Initial experiments were carried out following a four-component reaction approach, according to the literature $[18,19]$, through a simultaneous mixing of a 2-bromoketone, an 
ammonia source, $\mathrm{NaSH}$, and a ketone under diverse reaction conditions, which are summarized in Scheme 1 and Table 1. Preliminary results represented in entries 1-3 showed poor yields of thiazoline, as a consequence of incompatibility of aqueous ammonia with the other components, especially 2-bromoketone, affording some degradation products related to competition in nucleophilic substitution on 2-bromoketones, which resulted in it being sensitive to ammonia. This behavior was kept with the introduction of other solvents (entries 4-7) at different temperatures (entries 8-10), as well as diverse ammonia sources (entries 11-18).

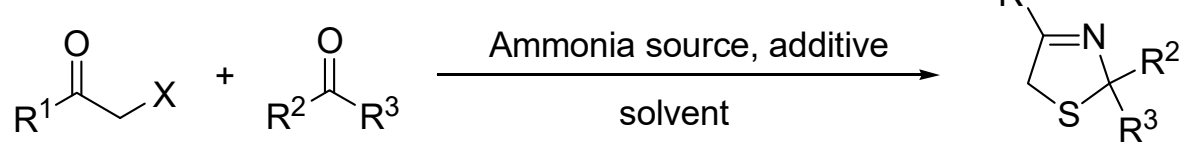

Scheme 1. Preparation of thiazolines from a four-component reaction.

Table 1. Synthesis of thiazoline 4 catalyzed by phenylacetylide 1 .

\begin{tabular}{|c|c|c|c|c|c|c|c|c|c|c|}
\hline Entry & $\mathbf{R} 1$ & $\mathbf{R 2}$ & R3 & $x$ & $\begin{array}{l}\text { Ammonia } \\
\text { Source }\end{array}$ & Solvent & Additive & $\begin{array}{c}\text { Reaction Tem- } \\
\text { perature }\left({ }^{\circ} \mathrm{C}\right)\end{array}$ & $\begin{array}{l}\text { Reaction } \\
\text { Time (h) }\end{array}$ & \%Yield \\
\hline 1 & $\mathrm{Me}$ & $\mathrm{Me}$ & $\mathrm{Me}$ & $\mathrm{Br}$ & $\mathrm{NH}{ }_{4} \mathrm{OH} / \mathrm{H}_{2} \mathrm{O}$ & - & $\mathrm{NaSH}$ & R. T. & 24 & $<5$ \\
\hline 2 & $\mathrm{Ph}$ & $\mathrm{Me}$ & $\mathrm{Me}$ & $\mathrm{Br}$ & $\mathrm{NH}_{4} \mathrm{OH} / \mathrm{H}_{2} \mathrm{O}$ & - & $\mathrm{NaSH}$ & R. T. & 24 & 0 \\
\hline 3 & $\mathrm{Me}$ & $\mathrm{Ph}$ & $\mathrm{Ph}$ & $\mathrm{Br}$ & $\mathrm{NH}_{4} \mathrm{OH} / \mathrm{H}_{2} \mathrm{O}$ & - & $\mathrm{NaSH}$ & R. T. & 24 & 0 \\
\hline 4 & $\mathrm{Me}$ & $\mathrm{Me}$ & $\mathrm{Me}$ & $\mathrm{Br}$ & $\mathrm{NH}_{4} \mathrm{OH} / \mathrm{H}_{2} \mathrm{O}$ & $\mathrm{MeOH}$ & $\mathrm{NaSH}$ & R. T. & 24 & 0 \\
\hline 5 & $\mathrm{Me}$ & $\mathrm{Me}$ & $\mathrm{Me}$ & $\mathrm{Br}$ & $\mathrm{NH}_{4} \mathrm{OH} / \mathrm{H}_{2} \mathrm{O}$ & Acetone & $\mathrm{NaSH}$ & R. T. & 24 & 0 \\
\hline 6 & $\mathrm{Me}$ & $\mathrm{Me}$ & $\mathrm{Me}$ & $\mathrm{Br}$ & $\mathrm{NH}_{4} \mathrm{OH} / \mathrm{H}_{2} \mathrm{O}$ & $\mathrm{CH}_{2} \mathrm{Cl}_{2}$ & $\mathrm{NaSH}$ & R. T. & 24 & 0 \\
\hline 7 & $\mathrm{Me}$ & $\mathrm{Me}$ & $\mathrm{Me}$ & $\mathrm{Br}$ & $\mathrm{NH}_{4} \mathrm{OH} / \mathrm{H}_{2} \mathrm{O}$ & THF & $\mathrm{NaSH}$ & R. T. & 24 & 0 \\
\hline 8 & $\mathrm{Me}$ & $\mathrm{Me}$ & $\mathrm{Me}$ & $\mathrm{Br}$ & $\mathrm{NH}_{4} \mathrm{OH} / \mathrm{H}_{2} \mathrm{O}$ & $\mathrm{MeOH}$ & $\mathrm{NaSH}$ & Reflux & 24 & 0 \\
\hline 9 & $\mathrm{Ph}$ & $\mathrm{Me}$ & $\mathrm{Me}$ & $\mathrm{Br}$ & $\mathrm{NH}_{4} \mathrm{OH} / \mathrm{H}_{2} \mathrm{O}$ & $\mathrm{MeOH}$ & $\mathrm{NaSH}$ & Reflux & 24 & 0 \\
\hline 10 & $\mathrm{Me}$ & $\mathrm{Ph}$ & $\mathrm{Ph}$ & $\mathrm{Br}$ & $\mathrm{NH}_{4} \mathrm{OH} / \mathrm{H}_{2} \mathrm{O}$ & $\mathrm{MeOH}$ & $\mathrm{NaSH}$ & Reflux & 24 & 0 \\
\hline 11 & $\mathrm{Ph}$ & $\mathrm{Me}$ & $\mathrm{Me}$ & $\mathrm{Br}$ & $\mathrm{NH}_{4} \mathrm{OAc}$ & Acetone & $\mathrm{NaSH}$ & R. T. & 48 & 0 \\
\hline 12 & $\mathrm{Me}$ & $\mathrm{Me}$ & $\mathrm{Me}$ & $\mathrm{Br}$ & $\mathrm{NH}_{4} \mathrm{OAc}$ & Acetone & $\mathrm{NaSH}$ & R. T. & 48 & 0 \\
\hline 13 & $\mathrm{Me}$ & $\mathrm{Me}$ & $\mathrm{Me}$ & $\mathrm{Br}$ & $\mathrm{NH}_{4} \mathrm{OAc}$ & $\mathrm{MeOH}$ & $\mathrm{NaSH}$ & R. T. & 48 & 0 \\
\hline 14 & $\mathrm{Ph}$ & $\mathrm{Me}$ & $\mathrm{Me}$ & $\mathrm{Br}$ & $\mathrm{NH}_{4} \mathrm{OAc}$ & $\mathrm{MeOH}$ & $\mathrm{NaSH}$ & R. T. & 48 & 0 \\
\hline 15 & $\mathrm{Ph}$ & $\mathrm{Me}$ & $\mathrm{Me}$ & $\mathrm{Br}$ & $\mathrm{NH}_{4} \mathrm{OAc}$ & $\mathrm{MeOH}$ & $\mathrm{NaSH}$ & Reflux & 24 & 0 \\
\hline 16 & $\mathrm{Me}$ & $\mathrm{Ph}$ & $\mathrm{Ph}$ & $\mathrm{Br}$ & $\mathrm{NH}_{4} \mathrm{OAc}$ & $\mathrm{AcOH}$ & $\mathrm{NaSH}$ & 100 & 24 & 0 \\
\hline 17 & $\mathrm{Me}$ & $\mathrm{Ph}$ & $\mathrm{Ph}$ & $\mathrm{Br}$ & $\mathrm{NH}_{4} \mathrm{OAc}$ & DMF & $\mathrm{NaSH}$ & 100 & 24 & 0 \\
\hline 18 & $\mathrm{Me}$ & $\mathrm{Ph}$ & $\mathrm{Ph}$ & $\mathrm{Br}$ & $\mathrm{NH}_{4} \mathrm{OAc}$ & Toluene & $\mathrm{NaSH}$ & 100 & 24 & 0 \\
\hline 19 & $\mathrm{Me}$ & $4-\mathrm{ClC}_{6} \mathrm{H}_{4}$ & $\mathrm{H}$ & $\mathrm{Br}$ & $\mathrm{NH}_{4} \mathrm{OH} / \mathrm{H}_{2} \mathrm{O}$ & - & $\mathrm{NaSH}$ & R. T. & 24 & 8 \\
\hline 20 & $\mathrm{Me}$ & $4-\mathrm{ClC}_{6} \mathrm{H}_{4}$ & $\mathrm{H}$ & $\mathrm{Br}$ & $\mathrm{NH}{ }_{4} \mathrm{OH} / \mathrm{H}_{2} \mathrm{O}$ & $\mathrm{MeOH}$ & $\mathrm{NaSH}$ & R. T. & 24 & 12 \\
\hline 21 & $\mathrm{Me}$ & $4-\mathrm{ClC}_{6} \mathrm{H}_{4}$ & $\mathrm{H}$ & $\mathrm{Br}$ & $\mathrm{NH}_{4} \mathrm{OH} / \mathrm{H}_{2} \mathrm{O}$ & $\mathrm{MeOH}$ & $\mathrm{NaSH}$ & R. T. & 24 & $<5$ \\
\hline 22 & $\mathrm{Me}$ & $4-\mathrm{ClC}_{6} \mathrm{H}_{4}$ & $\mathrm{H}$ & $\mathrm{Br}$ & $\mathrm{NH}_{3} / \mathrm{MeOH}$ & $\mathrm{MeOH}$ & $\mathrm{NaSH}$ & R. T. & 24 & 20 \\
\hline 23 & $\mathrm{Me}$ & $4-\mathrm{ClC}_{6} \mathrm{H}_{4}$ & $\mathrm{H}$ & $\mathrm{Br}$ & $\mathrm{NH}_{3} / \mathrm{MeOH}$ & $\mathrm{MeOH}$ & $\begin{array}{l}\mathrm{NaSH}, \\
\mathrm{MgSO}_{4}\end{array}$ & R. T. & 24 & 35 \\
\hline 24 & $\mathrm{Me}$ & $4-\mathrm{ClC}_{6} \mathrm{H}_{4}$ & $\mathrm{H}$ & $\mathrm{SH}$ & $\mathrm{NH}_{3} / \mathrm{MeOH}$ & $\mathrm{MeOH}$ & $\mathrm{MgSO}_{4}$ & R. T. & 24 & 64 \\
\hline 25 & $\mathrm{Me}$ & cyclohexan & & $\mathrm{SH}$ & $\mathrm{NH}_{3} / \mathrm{MeOH}$ & $\mathrm{MeOH}$ & $\mathrm{MgSO}_{4}$ & R. T. & 24 & 68 \\
\hline
\end{tabular}

The change of a ketone by an aldehyde in Asinger Reaction slightly improved thiazoline yields up to $20 \%$, using the ammonia solution in methanol described by Silvani et al. [14]. According to these authors, anhydrous conditions using $\mathrm{MgSO}_{4}$ also contributed to yield optimization, however, a radical change in reaction yield was observed when 1-mercaptopropan-2-one was used instead of the 2-bromoketone/ $\mathrm{NaSH}$ system, avoiding secondary nucleophilic substitutions. This reactivity pattern was corroborated through the use of cyclohexanone giving the corresponding thiazoline in $68 \%$ yield. Hence, the components reduction in the Asinger reaction was compensated by a substantial yield increase.

From these improvements, a general three-component synthetic protocol was developed via straightforward reaction of 1-mercapto propan-2-one and ammonia/MeOH, and was modulated by an aldehyde (Scheme 2). Results in Table 2 show the procedure scope covering distinct substituents in the aldehyde component, giving yields ranged from $33 \%$ to $90 \%$. 


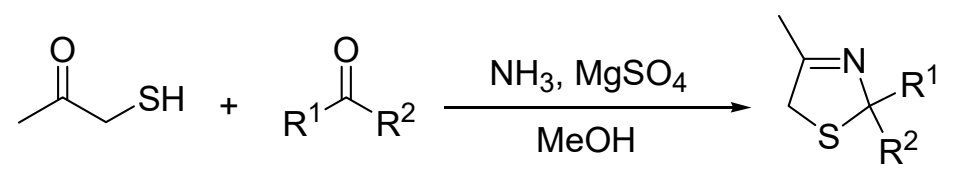

Scheme 2. Preparation of thiazolines from a three-component reaction at room temperature and under microwave irradiation.

Table 2. Thiazoline yields obtained at room temperature and under microwave irradiation.

\begin{tabular}{ccccc}
\hline Compound & $\mathbf{R}^{1}$ & $\mathbf{R}^{\mathbf{2}}$ & \%Yield at R.T. & \%Yield under MW \\
\hline $\mathbf{1}$ & $4-\mathrm{ClC}_{6} \mathrm{H}_{4}$ & $\mathrm{H}$ & 64 & 71 \\
$\mathbf{2}$ & $2-\mathrm{ClC}_{6} \mathrm{H}_{4}$ & $\mathrm{H}$ & 77 & 47 \\
$\mathbf{3}$ & $\mathrm{Ph}$ & $\mathrm{H}$ & 33 & 50 \\
$\mathbf{4}$ & $4-\mathrm{OHC}_{6} \mathrm{H}_{4}$ & $\mathrm{H}$ & 55 & 65 \\
$\mathbf{5}$ & $4-\mathrm{CH}_{3} \mathrm{C}_{6} \mathrm{H}_{4}$ & $\mathrm{H}$ & 69 & 49 \\
$\mathbf{6}$ & $2,3-\left(\mathrm{OCH}_{3}\right)_{2} \mathrm{C}_{6} \mathrm{H}_{3}$ & $\mathrm{H}$ & 69 & 75 \\
$\mathbf{7}$ & $\left(3-\mathrm{OCH}_{3}-4-\mathrm{OH}\right) \mathrm{C}_{6} \mathrm{H}_{3}$ & $\mathrm{H}$ & 90 & 70 \\
$\mathbf{8}$ & $\left(2-\mathrm{OCH}_{2} \mathrm{C} \equiv \mathrm{CH}\right) \mathrm{C}_{6} \mathrm{H}_{4}$ & $\mathrm{H}$ & 88 & 73 \\
$\mathbf{9}$ & cyclohexanone & & 68 & 75 \\
\hline
\end{tabular}

Based on our previous experiences on microwave-assisted reactions [20], we considered that these conditions could be extended to the Asinger reaction. Thus, early investigations revealed thiazoline formation from the last studied three-component reaction treated under microwave irradiation. The best yields were obtained at $40{ }^{\circ} \mathrm{C}(200 \mathrm{~W})$, which is comparable to, and in some cases higher than those observed at room temperature (see Table 2), but showed shorter reaction times. To the best of our knowledge, this was the first report about the synthesis of thiazolines from Asinger reaction using microwave irradiation as an alternative energy source.

The above examples demonstrate the feasibility of this methodology, and future studies are envisioned to broaden the applications of thiazolines in the synthesis of biologically active molecules, potential drugs, and ligands for catalysis.

\section{Experiment}

The starting materials were purchased from Aldrich Chemical Co. and were used without further purification. Solvents were distilled before use. Silica plates of $0.20 \mathrm{~mm}$ thickness were used for thin layer chromatography. The melting points were determined with a Krüss Optronic melting point apparatus and they were uncorrected. ${ }^{1} \mathrm{H}$ and ${ }^{13} \mathrm{C}$ NMR spectra were recorded using a Bruker Avance 300-MHz; the chemical shifts $(\delta)$ are given in ppm relative to TMS as the internal standard (0.00). For analytical purposes, the mass spectra were recorded on a Shimadzu GCMS-QP2010 Plus in the EI mode, $70 \mathrm{eV}$, $200{ }^{\circ} \mathrm{C}$ via direct inlet probe. Only the molecular and parent ions $(\mathrm{m} / \mathrm{z})$ were reported. IR spectra were recorded on a Bruker Tensor 27 equipment. The microwave-assisted reactions were performed using a CEM Discover microwave unit (constant factor of the microwave 1.214). The temperature was monitored with an IR temperature sensor. In all experiments, the microwave temperature was held constant. Reactions were carried out in 5-mL glass vessels, which were sealed with a cap septum. The specific reaction time corresponded to the total irradiation time.

\subsection{Synthesis of Thiazolines under Conventional Conditions-General Procedure}

The appropriate aldehyde or ketone $(1 \mathrm{mmol})$ and anhydrous $\mathrm{MgSO}_{4}(0.360 \mathrm{~g}, 3$ $\mathrm{mmol}$ ) were added to a $\sim 13 \mathrm{M}$ ammonia solution in $\mathrm{MeOH}(1 \mathrm{~mL})$, and the mixture was stirred for $1 \mathrm{~h}$ at room temperature. Mercaptoacetone $(0.099 \mathrm{~g}, 1.1 \mathrm{mmol})$ was added dropwise, over $5 \mathrm{~min}$. The resulting mixture was stirred for $24 \mathrm{~h}$ at room temperature. The mixture was filtered through celite. The solvent was removed under reduced pressure and the final product was purified by column chromatography $\left(\mathrm{SiO}_{2}\right.$, hexane/AcOEt 8:2). 


\subsection{Synthesis of Thiazolines under Microwave Irradiation Conditions-General Procedure}

A 5-mL microwave vial was charged with the corresponding aldehyde or ketone (1 mmol), $\mathrm{MgSO}_{4}(0.360 \mathrm{~g}, 3 \mathrm{mmol})$ and a $13 \mathrm{M}$ ammonia solution in $\mathrm{MeOH}(1 \mathrm{~mL})$ and a cylindrical magnetic stirring bar. The vessel was sealed with a septum, placed into the microwave cavity of a CEM Discover microwave unit and irradiated to heat the reaction mixture at $40{ }^{\circ} \mathrm{C}$. The total heating time was $10 \mathrm{~min}$ at $200 \mathrm{~W}, 40^{\circ} \mathrm{C}$. After the first cycle, mercaptoacetone $(0.099 \mathrm{~g}, 1.1 \mathrm{mmol})$ was added and the mixture was irradiated with same parameters for $10 \mathrm{~min}$. When the reaction was completed, the vial was cooled to room temperature. The vial was then opened and the mixture was filtered through celite. The solvent was removed under reduced pressure and the final product was purified by column chromatography $\left(\mathrm{SiO}_{2}\right.$, hexane/AcOEt 8:2).

\subsection{2-(4-Chlorophenyl)-4-methyl-2,5-dihydrothiazole 1}

4-Chlorobenzaldehyde and 1-mercaptopropan-2-one afforded 2-(4-chlorophenyl)-4-methyl-2,5-dihydrothiazole 1 as white solid. Yields: $135.0 \mathrm{mg}$ (64\% at room temperature conditions) and $149.8 \mathrm{mg}$ (71\% under microwave conditions). IR (ATR) vmax 2922, 1489, 1089, $828 \mathrm{~cm}^{-1} .{ }^{1} \mathrm{H}$ NMR $\left(300 \mathrm{MHz}, \mathrm{CDCl}_{3}\right) \delta 7.3(\mathrm{~d}, J=6 \mathrm{~Hz}, 2 \mathrm{H}), 7.2(\mathrm{~d}, J=6 \mathrm{~Hz}, 2 \mathrm{H}) 6.56(\mathrm{~s}$, $1 \mathrm{H}), 4.09(\mathrm{dd}, J=5 \mathrm{~Hz}, J=16 \mathrm{~Hz}, 1 \mathrm{H}), 3.98(\mathrm{dd}, J=3 \mathrm{~Hz}, J=16 \mathrm{~Hz}, 1 \mathrm{H}), 2.23(\mathrm{~s}, 3 \mathrm{H}) .{ }^{13} \mathrm{C}$ NMR $\left(75 \mathrm{MHz}, \mathrm{CDCl}_{3}\right) \delta 170.8,140.5,133.6,128.7,128.2,83.8,47.3,19.5$. MS [EI+] m/z (\%): $211[\mathrm{M}]^{+}(10)$.

\subsection{2-(2-Chlorophenyl)-4-methyl-2,5-dihydrothiazole 2}

2-Chlorobenzaldehyde and 1-mercaptopropan-2-one afforded 2-(2-chlorophenyl)-4-methyl-2,5-dihydrothiazole 2 as the white solid. Yields: $162.4 \mathrm{mg}$ (77\% at room temperature conditions) and $99.2 \mathrm{mg}$ (47\% under microwave conditions). IR (ATR) vmax 2916, 1469, 1036, $740 \mathrm{~cm}^{-1}$. ${ }^{1} \mathrm{H}$ NMR (300 MHz, $\left.\mathrm{CDCl}_{3}\right)$ 8 7.37-7.17 (m, 4H), 6.9 (s, 1H), $3.89(\mathrm{~s}, 2 \mathrm{H}), 2.25$ (s, 3H); ${ }^{13} \mathrm{C} \mathrm{NMR} \mathrm{(75} \mathrm{MHz,} \mathrm{CDCl}_{3}$ ) $\delta$ 172.2, 140.0, 132.3, 129.4, 128.8, 127.4, 127.1, 81.0, 46.6, 19.7. $\mathrm{MS}\left(\mathrm{EI}^{+}\right) \mathrm{m} / \mathrm{z}(\%): 211[\mathrm{M}]^{+}(5)$.

\subsection{4-Methyl-2-phenyl-2,5-dihydrothiazole 3}

Benzaldehyde and 1-mercaptopropan-2-one afforded 4-methyl-2-phenyl -2,5-dihydrothiazole 3 as white solid. Yields: $58.4 \mathrm{mg}$ ( $33 \%$ at room temperature conditions) and $88.5 \mathrm{mg}$ (50\% under microwave conditions). IR (ATR) vmax 2915, 1662, $1421 \mathrm{~cm}^{-1} .{ }^{1} \mathrm{H}$ NMR $\left(300 \mathrm{MHz}, \mathrm{CDCl}_{3}\right) \delta$ 7.42-7.25 (m, 5H), $6.62(\mathrm{bs}, 1 \mathrm{H}), 4.10(\mathrm{dd}, J=5 \mathrm{~Hz} J=15 \mathrm{~Hz}, 1 \mathrm{H}), 3.98$ $(\mathrm{dd}, J=3 \mathrm{~Hz}, J=15 \mathrm{~Hz}, 1 \mathrm{H}), 2.23(\mathrm{~s}, 3 \mathrm{H}) ;{ }^{13} \mathrm{C} \mathrm{NMR}\left(75 \mathrm{MHz}, \mathrm{CDCl}_{3}\right) \delta$ 170.3, 141.9, 128.6, 128.41, 127.9, 126.8, 84.5, 47.2, 19.5. $\left.\mathrm{MS}_{(\mathrm{EI}}{ }^{+}\right) \mathrm{m} / \mathrm{z}(\%): 177[\mathrm{M}]^{+}(15)$.

\subsection{4-(4-Methyl-2,5-dihydrothiazol-2-yl)phenol 4}

4-Hydroxybenzaldehyde and 1-mercaptopropan-2-one afforded 4-(4-methyl-2,5-dihydrothiazol-2-yl)phenol 4 as white solid. Yields: $106.1 \mathrm{mg}$ (55\% at room temperature conditions) and $125.4 \mathrm{mg}$ (65\% under microwave conditions). IR (ATR) vmax 3065, 2900, 1663, $1514 \mathrm{~cm}^{-1}$. ${ }^{1} \mathrm{H}$ NMR $\left(300 \mathrm{MHz}, \mathrm{CDCl}_{3}\right) 8.69$ (bs, $\left.1 \mathrm{H},-\mathrm{OH}\right), 7.12(\mathrm{~d}, J=9 \mathrm{~Hz}, 2 \mathrm{H}), 6.79(\mathrm{~d}, J=9 \mathrm{~Hz}), 6.53$ (bs, $1 \mathrm{H}), 4.10(\mathrm{dd}, J=5 \mathrm{~Hz}, J=16 \mathrm{~Hz}, 1 \mathrm{H}), 3.96(\mathrm{dd}, J=3 \mathrm{~Hz}, J=16 \mathrm{~Hz}, 1 \mathrm{H}) 2.22(\mathrm{~s}, 3 \mathrm{H}) ;{ }^{13} \mathrm{C}$ NMR (75 MHz, $\left.\mathrm{CDCl}_{3}\right) \delta 169.6,156.9,132.6,127.9,115.48,84.3,47.0,19.4 . \mathrm{MS}\left(\mathrm{EI}^{+}\right) \mathrm{m} / \mathrm{z}(\%)$ : $193[\mathrm{M}]^{+}(5)$.

\subsection{4-Methyl-2-(p-tolyl)-2,5-dihydrothiazole 5}

4-Methylbenzaldehyde and 1-mercaptopropan-2-one afforded 4-methyl-2-( $p$-tolyl)-2,5-dihydrothiazole 5 as white solid. Yields: $131.7 \mathrm{mg}$ (69\% at room temperature conditions) and 93.5 mg (49\% under microwave conditions). IR (ATR) vmax 2916, 1662, $817 \mathrm{~cm}^{-1}$. ${ }^{1} \mathrm{H}$ NMR $\left(300 \mathrm{MHz}, \mathrm{CDCl}_{3}\right) \delta 7.19(\mathrm{~d}, J=9 \mathrm{~Hz}, 2 \mathrm{H}), 7.13(\mathrm{~d}, J=9 \mathrm{~Hz}, 2 \mathrm{H}), 6.59(\mathrm{bs}, 1 \mathrm{H}), 4.11(\mathrm{dd}, J=6 \mathrm{~Hz}$, $J=15 \mathrm{~Hz}, 1 \mathrm{H}) 3.98(\mathrm{dd}, J=6 \mathrm{~Hz}, J=15 \mathrm{~Hz}, 1 \mathrm{H}), 2.33(\mathrm{~s}, 3 \mathrm{H}) 2.24(\mathrm{~s}, 3 \mathrm{H}) ;{ }^{13} \mathrm{C} \mathrm{NMR}(75 \mathrm{MHz}$, $\left.\mathrm{CDCl}_{3}\right) \delta$ 169.7, 138.7, 137.3, 128.9, 126.3, 84.1, 46.9, 29.4, 20.8, 19.2. MS (EI+) m/z (\%): 191 $[\mathrm{M}]^{+}(10)$. 


\subsection{2-(2,3-Dimethoxyphenyl)-4-methyl-2,5-dihydrothiazole 6}

2,3-Dimethox-benzaldehyde and 1-mercaptopropan-2-one afforded 2-(2,3-dimethoxyphenyl)-4-methyl-2,5-dihydrothiazole 6 as white solid. Yields: $163.5 \mathrm{mg}$ (69\% at room temperature conditions) and $177.7 \mathrm{mg}$ (75\% under microwave conditions). IR (ATR) vmax 2935, 2834, 1665, 1478, $1263 \mathrm{~cm}^{-1} .{ }^{1} \mathrm{H}$ NMR $\left(300 \mathrm{MHz}, \mathrm{CDCl}_{3}\right) \delta 7.02(\mathrm{dd}, J=9 \mathrm{~Hz}, J=6 \mathrm{~Hz}$, $1 \mathrm{H}), 6.94(\mathrm{bs}, 1 \mathrm{H}), 6.84(\mathrm{dd}, J=3 \mathrm{~Hz}, J=6 \mathrm{~Hz}, 1 \mathrm{H}), 6.76(\mathrm{dd}, J=3 \mathrm{~Hz}, J=6 \mathrm{~Hz}, 1 \mathrm{H}), 4.05$ $(\mathrm{dd}, J=6 \mathrm{~Hz}, J=15 \mathrm{~Hz}, 1 \mathrm{H}) 3.98(\mathrm{dd}, J=6 \mathrm{~Hz}, J=15 \mathrm{~Hz}, 1 \mathrm{H}), 3.91(\mathrm{~s}, 3 \mathrm{H}), 3.86(\mathrm{~s}, 3 \mathrm{H}), 2.26$ (s, 3H); ${ }^{13} \mathrm{C}$ NMR (75 MHz, $\mathrm{CDCl}_{3}$ ) \& 169.2, 150.9, 144.3, 134.6, 122.5, 117.0, 110.3, 59.4, 54.2, 45.2, 18.1. MS (EI+) m/z (\%): $237[\mathrm{M}]^{+}(20)$.

\subsection{2-Methoxy-4-(4-methyl-2,5-dihydrothiazol-2-yl)phenol 7}

4-Hydroxy-3-methoxy-benzaldehyde and 1-mercaptopropan-2-one afforded 2-methoxy4-(4-methyl-2,5-dihydrothiazol-2-yl)phenol 7 as white solid. Yields: $200.7 \mathrm{mg}$ (90\% at room temperature conditions) and $156.1 \mathrm{mg}$ (70\% under microwave conditions). IR (ATR) vmax 3101, 2920, 1595, 1368, $1270 \mathrm{~cm}^{-1} .{ }^{1} \mathrm{H}$ NMR $\left(300 \mathrm{MHz}, \mathrm{CDCl}_{3}\right) \delta 6.85-6.82(\mathrm{~m}, 3 \mathrm{H})$, $6.55(\mathrm{bs}, 1 \mathrm{H}), 5.73(\mathrm{bs}, 1 \mathrm{H}), 4.11(\mathrm{dd}, J=3 \mathrm{~Hz}, J=16 \mathrm{~Hz}, 1 \mathrm{H}), 3.98(\mathrm{dd}, J=3 \mathrm{~Hz}, J=16 \mathrm{~Hz}$, $1 \mathrm{H}), 3.88(\mathrm{~s}, 3 \mathrm{H}), 2.24(\mathrm{~s}, 3 \mathrm{H}) ;{ }^{13} \mathrm{C}$ NMR $\left(75 \mathrm{MHz}, \mathrm{CDCl}_{3}\right) \delta$ 170.0, 146.5, 145.4, 133.7, 119.9, 114.3, 109.4, 84.5, 55.9, 47.2, 19.6. MS (EI+) m/z (\%): 223 [M]

\subsection{4-Methyl-2-(2-(prop-2-yn-1-yloxy)phenyl)-2,5-dihydrothiazole 8}

2-Prop-2-ynyloxy-benzaldehyde and 1-mercaptopropan-2-one afforded 4-methyl-2-(2(prop-2-yn-1-yloxy)phenyl)-2,5-dihydrothiazole 8 as white solid. Yields: $203.2 \mathrm{mg}$ (88\% at room temperature conditions) and $168.3 \mathrm{mg}$ (73\% under microwave conditions). IR (ATR) vmax 3285, 2918, 2120, 1663, 1487, 1220, $751 \mathrm{~cm}^{-1} .{ }^{1} \mathrm{H}$ NMR $\left(300 \mathrm{MHz}, \mathrm{CDCl}_{3}\right) \delta$ 8.25-7.23 $(\mathrm{m}, 1 \mathrm{H}), 7.21-7.19(\mathrm{~m}, 1 \mathrm{H}) 6.99-6.95(\mathrm{~m}, 1 \mathrm{H}), 6.93(\mathrm{bs}, 1 \mathrm{H}), 4.75(\mathrm{~s}, 2 \mathrm{H}) 4.03(\mathrm{dd}, J=3 \mathrm{~Hz}$, $J=9 \mathrm{~Hz}, 1 \mathrm{H}), 3.94(\mathrm{dd}, J=1.5 \mathrm{~Hz}, J=9 \mathrm{~Hz}, 1 \mathrm{H}), 2.52(\mathrm{~s}, 1 \mathrm{H}) 2.26(\mathrm{~s}, 3 \mathrm{H}) .{ }^{13} \mathrm{C}$ NMR $(75 \mathrm{MHz}$, $\left.\mathrm{CDCl}_{3}\right) \delta$ 154.1, 131.6, 128.6, 126.8, 121.6, 112.0, 78.8, 78.5, 75.5, 56.2, 46.4, 19.7. MS [EI+] m/z (\%): $231[\mathrm{M}]^{+}(5)$.

\subsection{3-Methyl-1-thia-4-azaspiro[4.5]dec-3-ene 9}

Cyclohexanone and 1-mercaptopropan-2-one afforded 3-methyl-1-thia-4-azaspiro[4.5] dec-3-ene 9 as white solid. Yields: $114.9 \mathrm{mg}$ ( $68 \%$ at room temperature conditions) and $126.7 \mathrm{mg}$ (75\% under microwave conditions). IR (ATR) vmax 2927, 1691, 1444, $894 \mathrm{~cm}^{-1}$. ${ }^{1} \mathrm{H}$ NMR $\left(300 \mathrm{MHz}, \mathrm{CDCl}_{3}\right) \delta 3.77(\mathrm{~s}, 2 \mathrm{H}), 2.10(\mathrm{~s}, 3 \mathrm{H}), 2.04-1.16(\mathrm{~m}, 10 \mathrm{H}) .{ }^{13} \mathrm{C}$ NMR (75 MHz, $\left.\mathrm{CDCl}_{3}\right) \delta$ 165.7, 95.4, 44.3, 40.9, 24.8, 24.1, 19.8. MS [EI+] m/z (\%): 169 [M] ${ }^{+}(10)$.

\section{Conclusions}

Thiazolines are readily available from microwave assisted Asinger reaction through a synthetic procedure that takes advantage of joining both, a multicomponent reaction approach and microwave irradiation methods. The simplicity of this methodology suggests that this route to thiazolines can have widespread application.

Author Contributions: Conceptualization, E.C.-Y.; methodology, R.E.G.-C., L.G.-R., M.C.-R., and N.Z.-S.; formal analysis, N.Z.-S.; investigation, R.E.G.-C., N.Z.-S., E.C.-Y., B.A.F.-U., M.A.G.-E., and M.V.B.U.; resources, B.A.F.-U. and E.C.-Y.; data curation, N.Z.-S., M.A.G.-E., and M.V.B.U.; writing-original draft preparation, E.C.-Y.; writing - review and editing, E.C.-Y.; supervision, E.C.-Y., M.A.G.-E., and M.V.B.U.; project administration, E.C.-Y.; funding acquisition, B.A.F.-U. and E.C.-Y. All authors have read and agreed to the published version of the manuscript.

Funding: This research was funded by CONACYT-Mexico, project No. A1-S-18230 and fellowship for R.E.G.C., CVU: 229559.

Acknowledgments: Financial support from CONACYT is gratefully acknowledged. The authors would like to thank A. Nuñez, L. Triana and M. C. Martínez for the technical support.

Conflicts of Interest: The authors declare no conflict of interest. 


\section{References}

1. Gaumont, A.C.; Gulea, M.; Levillain, J. Overview of the Chemistry of 2-Thiazolines. Chem. Rev. 2009, 109, 1371-1401.

2. Alom, N.E.; Wu, F.; Li, W. One-Pot Strategy for Thiazoline Synthesis from Alkenes and Thioamides. Org. Lett. 2017, 19, 930933.

3. Alsharif, Z.A.; Alam, M. Modular synthesis of thiazoline and thiazole derivatives by using a cascade protocol. RSC Adv. 2017, 7, 32647.

4. Ahmad, A.; Ahmad, A.; Sudhakar, R.; Varshney, H.; Subbarao, N.; Ansari, S.; Rauf, A.; Khan, A.U. Designing, synthesis and antimicrobial action of oxazoline and thiazoline derivatives of fatty acid esters. J. Biomol. Struct. Dyn. 2017, 35, 3412-3431.

5. Oniga, O.; Ndongo, J.T.; Moldovan, C.; Tiperciuc, B.; Oniga, S.; Pîrnău, A.; Vlase, L.; Verité, P. Synthesis and antimicrobial activity of some new 2-hydrazone-thiazoline-4-ones. Farmacia 2012, 60, 785- 797.

6. You, S.L.; Razavi, H.; Kelly, J.W. A Biomimetic Synthesis of Thiazolines Using Hexaphenyloxodiphosphonium Trifluoromethanesulfonate. Angew. Chem. Int. Ed. 2003, 42, 83-85.

7. Schneider, J.M.F.M.; Sales, E.S.; Livotto, P.R.; Schneider, P.H.; Merlo, A.A. Synthesis of New Family of Thiazoline and Thiazole Esters and Investigation of their Thermal Properties. J. Braz. Chem. Soc. 2014, 25, 1493-1503.

8. Diness, F.; Nielsen, D.S.; Fairlie, D.P. Synthesis of the Thiazole-Thiazoline Fragment of Largazole Analogues. J. Org. Chem. 2011, 76, 9845-9851.

9. Chen, J.; Forsyth, C.J. Total synthesis of the marine cyanobacterial cyclodepsipeptide apratoxin A. Proc. Natl. Acad. Sci. USA 2004, 101, 12067-12072.

10. Asinger, F. Über die gemeinsame Einwirkung von Schwefel und Ammoniak auf Ketone. Angew. Chem. 1956, 68, 413.

11. Asinger, F. Chemiker-Treffen Salzburg. Angew. Chem. 1956, 68, 377.

12. Asinger, F.; Thiel, M.; Pallas, E. Die gemeinsame einwirkung von schwefel und ammoniak auf diathylketon. Liebigs Ann. Chem. 1957, 602, 37-49.

13. Schlüter, T.; Frerichs, N.; Schmidtmann, M.; Martens, J. Consecutive Multicomponent Reactions: Synthesis of 3-Acyl-4-alkynylSubstituted 1,3-Thiazolidines. Synthesis 2018, 50, 1123-1132.

14. Rainoldi, G.; Begnini, F.; Silvani, A.; Lesma, G. Efficient Synthesis of Spirooxindole-Fused 3-Thiazoline Derivatives by a OnePot Asinger-Type Reaction. Synlett 2016, 27, 2831-2835.

15. Brockmeyer, F.; Schoemaker, R.; Schmidtmann, M.; Martens, J. Multicomponent reaction for the first synthesis of 2,2-dialkyland 2-alkyl-2-aralkyl-5,6-diaryl-2H-1,3-thiazines as scaffolds for various 3,4-dihydro-2H-1,3-thiazine derivatives. Org. Biomol. Chem. 2014, 12, 292-299.

16. Brockmeyer, F.; van Gerven, D.; Saak, W.; Martens, J. Two Sequential Multicomponent Reactions: Synthesis of Thiazolidin-4yl-1,3,4-oxadiazoles under Mild Conditions. Synthesis 2014, 46, 1603-1612.

17. Zeinab Faghihi, Z.; Oskooie, H.A.; Heravi, M.M.; Tajbakhsh, M.; Shiri, M. A novel analogue of Asinger reaction for the synthesis of thiazinoquinoline derivatives. Monat. Chem. 2017, 148, 315-320.

18. Schlemminger, I.; Janknecht, H.H.; Maison, W.; Saak, W.; Martens, J. Synthesis of the First enantiomerically pure 3-thiazolines via Asinger reaction. Tetrahedron. Lett. 2000, 41, 7289-7292.

19. Asinger, F.; Offermanns, H. Syntheses with Ketones, Sulfur, and Ammonia or Amines at Room Temperature. Angew. Chem. Int. Ed. 1967, 6, 907-919.

20. García-Muñoz, A.; Ortega-Arizmendi, A.I.; García-Carrillo, M.A.; Díaz, E.; Gonzalez-Rivas, N.; Cuevas-Yañez, E. Direct, metalfree synthesis of benzyl alcohols and deuterated benzyl alcohols from p-toluenesulfonylhydrazones using water as solvent. Synthesis 2012, 44, 2237-2242. 